\title{
Toward Online Measurement of Decision State
}

\section{Joel Lachter, ${ }^{1,2}$ James C. Johnston, ${ }^{1}$ Greg S. Corrado ${ }^{3}$ \& James L McClelland ${ }^{3}$ ${ }^{1}$ NASA Ames Research Center, ${ }^{2}$ San Jose State University, ${ }^{3}$ Stanford University}

\section{A New Method}

\section{Goals}

Develop a behavioral measure of decision state that tracks the development of decisions over time

allows observers to indicate uncertainty with graded responses

Track influences of top-down and bottom-up information over time

\section{Task}

Observers determined which of two patches of dots had more dots. Observers were asked to indicate their confidence that more dots were on the right or left by positioning a small bar with a joystick. Observers were asked to respond as quickly as possible.

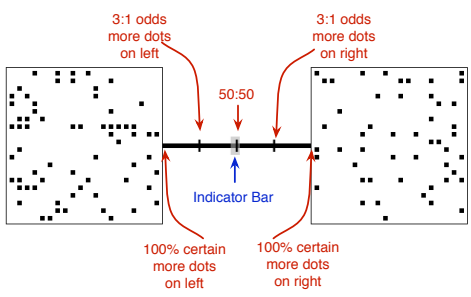

\section{Scoring}

Accuracy

Observers received points as a function of their distance from the correct response.

The function gave the highest expected value when observers accurately reported the probability they were correct.

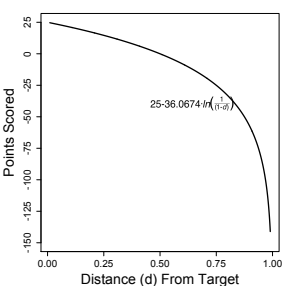

Speed

Trials ended at a random point in time, not controlled by the observer.

bservers received a score based on the bar position at the end of the trial. as quickly as possible when new information became available.
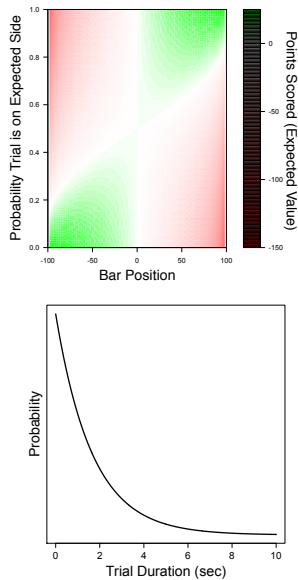

\section{Design}

We manipulated bottom-up discriminability across trials. One patch always had 50 dots, the other had 5I, 52, 54, 58 or 66

We manipulated top-down expectation across blocks. One patch was three times as likely to have more dots as the other.

Observers saw 10 blocks of 82 trials. The first 3 blocks of the session and the first 2 trials of every block were discarded. The remaining 80 trials/block contained 15 trials of each discriminability level on the expected side and 5 on the unexpected side.
Thus observers had to move the bar

\section{How well does the method work?}

\section{Overall Performance}

Performance reached asymptote after about 3 blocks (246 trials). Some observers performed much better than others. Almost hall or the observers scored fewer points than could have been
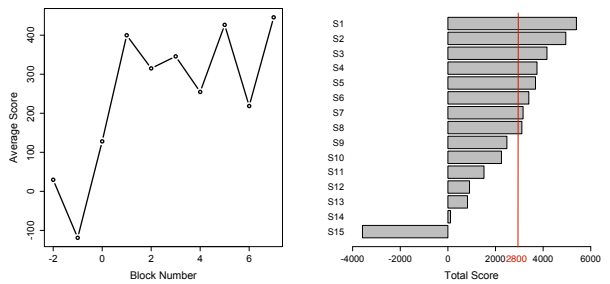

Reporting of Confidence No observers were close to optimal in the absence of perceptual information (e.g., before
stimulus onset). With perceptual information, all observers showed some degree of graded responding with the best obsevers reporting their confidence fairly accuraty.

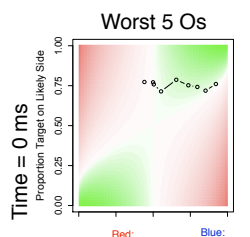

Middle 5 Os
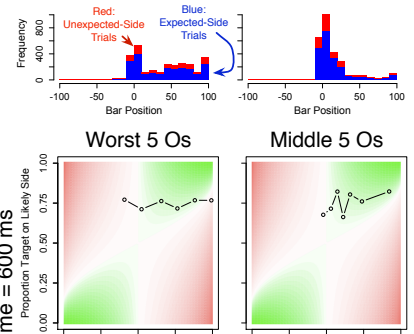

窟这.
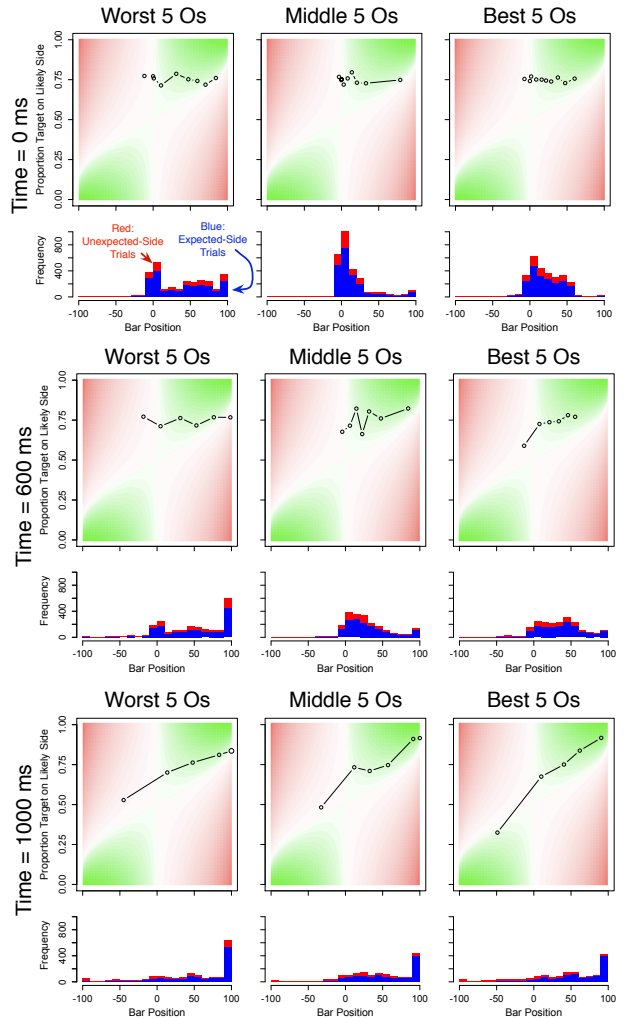

Middle 5 Os

Best 5 Os
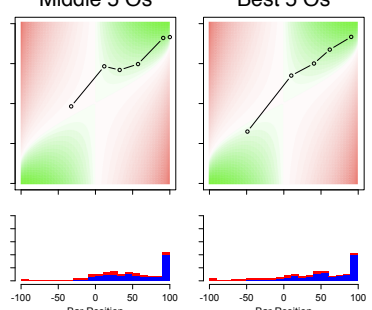

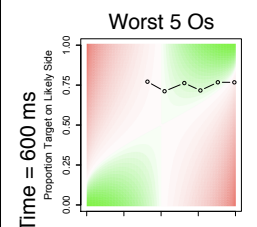

What can we learn using this method?

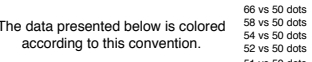

Looking at Individual Trials
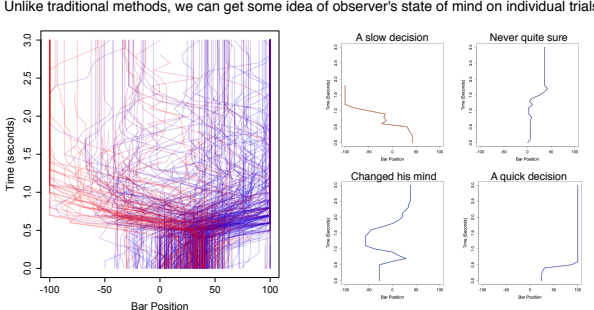

Examining patterns: How often do observers change their mind? If you find a particular data pattern of interest you can pull out the corresponding trials and
characterize them. Here we look at the first place the observer set the bar plotted against the
final bar position to find trials on which the observer changed their mind.

All Os, Trials $>1.5 \mathrm{sec}$
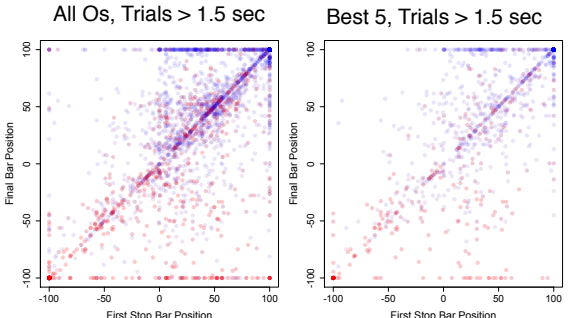

Expected and unexpected trials can be looked at independently.

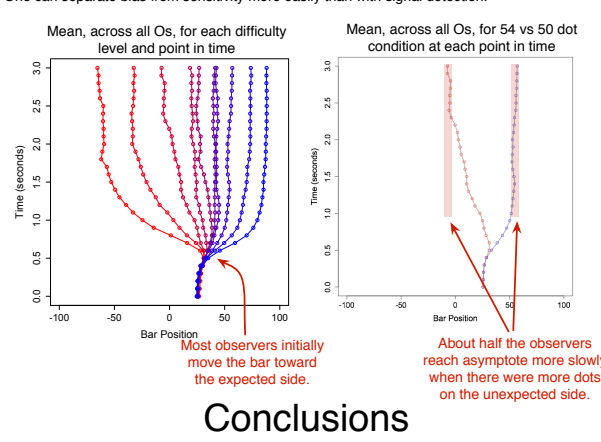

We have created a paradigm that allows us to capture the evolution of observer decision state can for all observers, track the time course of their decision probits that hey were correct, ability to examine single trials at a level of detail that is not captured with traditional psychophysical techniques. 